\title{
Calculation Method and Application of Inter-Regional and Inter- Provincial Market Trading Space for Promoting the Consumption of Clean Energy
}

\author{
Hao Tan ${ }^{1,2 *}$, Mingzhu Yuan ${ }^{1,2}$, Lei Wang ${ }^{1,2}$, Wujun Dong ${ }^{1,2}$, Ziyang Bai ${ }^{1,2}$ \\ ${ }^{1}$ NARI Group Corporation (State Grid Electric Power Research Institute), Nanjing, Jiangsu, 211000, China \\ ${ }^{2}$ Beijing Kedong Power Control System Co., Ltd., Beijing, Beijing, 100192, China
}

\begin{abstract}
Inter-provincial market trading is putting up with to implement the national energy strategy, increasing clean energy consuming and optimization energy allocation in large-scale. To promote energy consumption, the inter-province power transmission channel' potential needs to be full excavate to deal with clean energy fluctuation, improving trading frequency and reduce transaction period. This article puts up with an inter-province power market trading space calculation method to calculate the available power transmission ability of inter-provincial transmission channel, discovering transmission channel and releasing transaction space. Besides, for certain transmission channel or supply-demand province's electricity requirements, the calculation method can explore the trading market space, organizing clean energy evacuation to improve transmission channel utilization and safety checking pass rates, reducing blocking.
\end{abstract}

\section{Introduction}

In 2015, the government of China had published the Several Opinions on Further Deepening the Reform of Electric Power System (Zhongfa [2015] No. 9). As a result of the document, the construction of Chinese electricity market has been gradually accelerated. Up to now, the overall market framework of "unified market with two-level operation" has been basically established, including inter-regional and provincial power markets. The provincial power markets is mainly positioned to implement the national energy strategy, promote the consumption of clean energy and large-scale optimal allocation of energy resources. In 2019, the transactional electricity in the trans-provincial power market has reached 1061.9 billion $\mathrm{kWh}$, with clean energy accounting for $43.3 \%[2]$.

In order to effectively promote the absorption of clean energy and improve the efficiency of energy resource allocation, in view of the fluctuating and unpredictable characteristics of clean energy power generation, temporary transaction and emergency transaction will be organized according to the surplus power generation capacity of clean energy and the electricity purchase demand of each province when there is still surplus of trans-provincial transmission tie-lines.

At present, the inter-regional and inter-provincial power trading is organized by the Beijing Power Exchange Centre, and the trading result needs security check of the National Electric Power Control Centre. The safety check should be completed within the specified period, but when the safety check is not passed, the trading institutions should adjust the trading result and let the power dispatching organization check the result again until the safety check is passed. This iteration will greatly increase the organization cycle of power transaction. Therefore, when organizing clean energy inter-regional electricity transactions, considering the constraints of transmission tie-lines, limiting the amount of electricity traded will effectively improve the passing rate of security inspection, thus improving the efficiency of transaction organization.

According to the current inter-regional power market organization, the power dispatching organization shall timely provide the power trading organization with the available transfer capability (ATC) of each section and each path, as well as the distribution coefficient on different sections and paths [4]. Available transmission capacity is the remaining space in the transmission grid that can be used for market transactions. The calculation of available transmission capacity has been very mature after years of development and application, such as optimal power flow method [5], repeated power flow method [6] and probabilistic calculation [7].

The application of available transmission capacity in the inter-regional power market has just started. At present, the available transmission capacity is mainly used as the grid operation information publicly released in the information disclosure and provided to the market subjects as the reference for limittion. Application research in power trading mainly focuses on the available

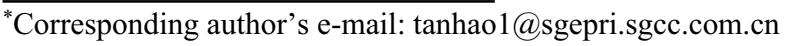


transmission capacity of trans-provincial transmission tie-lines [8-10]. In these studies, the available transmission capacity is taken as the constraint condition when the centralized transaction is cleared out, so as to reduce the impact of transaction organization on the security of the power grid, reduce unnecessary iterations in the process of security check, and improve the efficiency of transaction organization. In clearing calculation, it is a way to delimit the feasible area of transaction organization to take the available transmission capacity as the constraint. In the face of the inter-regional power trade that promotes the clean energy consumption, it is the key consideration to actively find the incremental trading space in advance, matching the fluctuation of clean energy output, and making full use of the residual capacity of transmission tie-line to organize inter-regional power trade. At present, the transaction path of inter-regional power transaction only specifies the relevant inter provincial transmission tie-line. The complete transaction path from sending end to receiving end has not been established. In order to effectively apply the available transmission capacity and distribution coefficient provided by power dispatching organization, it is necessary to build a simplified power grid model integrating transaction flow and power flow.

Starting from the simplified model of power grid based on available transmission capacity, this paper analyzes the available transmission capacity data of main tie-lines between provinces provided by power dispatching organization, finds the transmission tie-lines with transmission capacity release and their time periods, and calculates and analyzes the transaction space of inter provincial power market as the transaction organization the basis for determining the trading space.

\section{Available transmission capacity and simplified model of power grid}

\subsection{Available transmission capacity}

At present, the power grid operation data obtained by the trading center from the power dispatching organization include the available transmission capacity of each main tie-line and the distribution coefficient of transactions on different paths. The power grid operation data obtained by the trading center from the power dispatching organization include the available transmission capacity of each main tie-line and the distribution coefficient of transactions on different paths. Available transmission capacity (ATC), the Federal Energy Commission (FERC) of the United States gave the standard definition of ATC in 1996: ATC refers to the remaining commercial transmission capacity in the actual physical network on the basis of the existing transmission contract [11]. Available transmission capacity is defined as the maximum transmission power for commercial use between regions or provinces. The formula for calculating ATC is:

$$
A T C=T T C-T R M-E T C-C B M
$$

Among them, TTC is the total transfer capability, TRM is the transmission reliability margin, ETC is the existing transmission commitment, and CBM is the capacity benefit margin.

For different transaction cycles (such as annual, monthly, short-term within month) and time before the operation day, power dispatching organization have different transmission reliability margins for available transmission capacity, and with the shortening of transaction cycle and the approaching of operation day, the proportion of TRM is gradually reduced and updated [4].

\subsection{Simplified model of power network based on PTDF}

The calculation of ATC usually includes continuous current method, sensitivity analysis method, transfer distribution factor method, etc. The calculation of ATC for major tie-lines across provinces is usually based on DC power flow model, which reflects the impact of any transaction in the whole network on the available transmission capacity for a specific section through power transfer distribution factors (PTDF) [12]. The PTDF describes the change in the flow of the branches caused by the active power transmitted between the two regions [13], which can be expressed as:

$$
P T D F_{l . n}=\frac{\Delta P_{l}}{\Delta P_{n}}
$$

That is injecting power $\Delta P_{n}$ at node $\mathrm{n}$ will cause the power of line L changing $\Delta P_{l}$.

Therefore, for systems with $\mathrm{L}$ branches and $\mathrm{N}$ nodes:

$$
\begin{gathered}
\boldsymbol{P}_{L}=\boldsymbol{P T D F} \cdot \boldsymbol{P}_{N} \\
\boldsymbol{P T D F}=\left[\begin{array}{cccc}
P T D F_{11} & P T D F_{12} & \cdots & P T D F_{1 n} \\
P T D F_{11} & P T D F_{22} & \cdots & \cdots \\
\vdots & \vdots & \vdots & \vdots \\
P T D F_{l 1} & P T D F_{l 2} & \cdots & P T D F_{l n}
\end{array}\right]
\end{gathered}
$$

In the formula, $\boldsymbol{P}_{L}$ is the current of each branch of the system. PTDF is power transfer distribution factor matrix of the system. $\boldsymbol{P}_{N}$ is injection power of nodes.

Therefore, the maximum power that can be injected into the feeder node of a branch under the ATC constraint of the branch can be expressed as:

$$
P_{i n \text { max }}=\frac{P_{l}^{A T C}}{P T D F_{l}}
$$

Based on the ATC and distribution factors provided by the power dispatching organization for major interprovincial tie-lines/sections, the maximum transmission capacity for cross-provincial power transactions through designated tie-lines can be determined by formula (5). 


\subsection{Simplified model of power network with transaction flow and power flow}

Currently, the cross-provincial power transactions are organized according to the tie-line, that is, when the transaction organization specifies only one main crossprovincial contact line as the tie-line for this transaction. This tie-line concatenates the sending-end region with several participating provinces in the receiving-end region to form a transaction [14] from the sending-end province to the receiving-end province. However, the transaction space between the sending end and the receiving end determined by ATC of only a certain tieline on the path cannot reflect the actual transmission power, so it is necessary to establish a full power network model from the sending end to the receiving end. For the current cross-province power mid-term and longterm transactions, the calculation of power flow based on the whole network is very complex, and for long-term transactions, the results are still biased in the actual implementation and need to be adjusted several times. Therefore, the mid-term and long-term transactions do not need to carry out optimization clearing calculation of complex network power flow.

\section{Analysis of available transmission capacity}

\subsection{Acquisition of available transmission capacity data}

According to the way of data interaction between the current trading center and the power dispatching organization, the power dispatching organization updates the daily stability limit, available transmission capacity and allocation factor of the tie-line section contained in the operation area of the State Grid according to the operation of the power network, and then pushes them to the power trading center for the purpose of transaction organization and information disclosure. With the organization of transactions and the formation of power supply arrangements, the available transmission capacity decreases gradually, but due to the approaching of operation days, the transmission reliability margin decreases gradually. In addition, due to the adjustment of operation modes, changes in maintenance plans, the daily stability limit may also be adjusted. Therefore, the different versions of available transmission capacity and daily stability limit data pushed by power dispatching organization are compared and analyzed, the tielines/sections with changes in transaction space are found from the massive data, and incremental transactions are organized for the tie-lines/sections that release transaction space, and warnings are given for the tie-lines/sections that reduce transaction space according to the demand for power at the sending and receiving ends.

\subsection{The Evaluation Index of ATC}

Available transmission capacity and daily stability limit data pushed by power dispatching organization are time series of 24 points per day for a tie-line/section in the coming months. The analysis of available transmission capacity and daily stability limit data is to compare the data of the same tie-line / section and the same time period in two different versions of data. The consistency of two sets of time series data can be judged by the sum or mean of time series, but for available transmission capacity data or daily stability limit, there may be some cases where the sum is the same but the data is inconsistent: for example, for reasons such as maintenance time adjustment or contract replacement, only some segments of time series are exchanged equally, but the total amount is not changed, so it is impossible. Reflects changes in time series data. Therefore, this paper uses the evaluation index of regression model to compare and analyze time series data.

Common evaluation criteria include root mean square error (RMSE), mean square error (MSE), mean absolute percentage error (MAPE), R square (r-squared, R2), and so on.

The formula is shown in (6) (9):

$$
\begin{gathered}
\text { MSE }=\frac{1}{n} \sum_{i=1}^{n}\left(y^{(i)}-\hat{y}^{(i)}\right)^{2} \\
R M S E=\sqrt{\frac{1}{n} \sum_{i=1}^{n}\left(y^{(i)}-\hat{y}^{(i)}\right)^{2}} \\
M A P E=\sum_{i=1}^{n}\left|\frac{y^{(i)}-\hat{y}^{(i)}}{y^{(i)}}\right| \times \frac{100}{n} \\
R^{2}=1-\frac{\sum_{i}\left(\hat{y}^{(i)}-y^{(i)}\right)^{2}}{\sum_{i}\left(\bar{y}-y^{(i)}\right)^{2}}
\end{gathered}
$$

In those formulas, $n$ is time points for time series, $y^{(i)}$ is the value at point $i$ in historical data version, $\hat{y}^{(i)}$ is the value at point $i$ in current data version, $\bar{y}^{(i)}$ is the represents the average of the time series of historical data versions.

These indexes can effectively reflect the changes of time series data, even if there are adjustments of some time series segments but the sum is unchanged, the above indexes can make judgments. Among them, MSE is positively related to the error size, if the two versions of data are identical, $\mathrm{MSE}=0$, but the actual error size is difficult to quantify through this index; RMSE is similar to MSE, when RMSE $=0$, the two versions of data are identical, and the index is positively related to the error size; The MAPE is proportional to the actual error, MAPE $=0$ if the two versions of data are identical, and the R-squared is 1, indicating that the two time series are identical. The smaller R2, the larger the error, and it is difficult to quantify the actual error. The comparison of the centralized evaluation indexes is shown in Table 1. 
Table 1. Comparison of several evaluation indicators.

\begin{tabular}{llll}
\hline Index & $\begin{array}{l}\text { Non- } \\
\text { deviation }\end{array}$ & $\begin{array}{l}\text { With- } \\
\text { deviation }\end{array}$ & correlation \\
\hline$M S E$ & 0 & $(0,+\infty)$ & positive \\
$R M S E$ & 0 & $(0,+\infty)$ & positive \\
$M A P E$ & 0 & $(0,+\infty)$ & positive \\
$R^{2}$ & 1 & {$[0,1)$} & negative \\
\hline
\end{tabular}

If MAPE is selected as the measurement index, the change of data can be quantitatively reflected.

\subsection{Data comparison}

In order to effectively characterize the error size of the two versions of data, intuitively and quantitatively reflect the change of transaction space, this paper finally selects the average percentage error MAPE as the judgment index, which is proportional to the actual error, so the change of transaction space can be judged according to the actual value of the index. If the difference between the current version and the historical version is greater than 0 , the transaction space will increase, otherwise, the transaction space will decrease.

$$
\Delta=\sum_{i=1}^{n} \frac{\hat{y}^{(i)}-y^{(i)}}{n}
$$

Therefore, $\Delta$ and MAPE are comprehensively considered to calculate and analyze the available transmission capacity and daily stability limit data of the two versions. The and MAPE obtained by calculation are shown in Table 2.

Table 2. Judgment of transaction space variation.

\begin{tabular}{ccc}
\hline MAPE & $\Delta$ & Changes in trading space \\
\hline$>0$ & $>0$ & Increase \\
$>0$ & $<0$ & Decrease \\
$=0$ & $=0$ & No-change \\
$>0$ & $=0$ & Having exchange \\
\hline
\end{tabular}

\section{Analysis of trading space}

After analyzing the available transmission capacity and daily stability limit of trans provincial power market transmission tie-line, the tie-line / section with increased trading space within the specified time range can be found. For the provinces on both sides of the trading path of the tie-line or section, incremental trans provincial power trading can be organized according to the increased trading space. If there is only one transaction path connecting the two provinces, the transaction space between the provinces is equal to the maximum transmission power [15] of all the tie-lines / sections included in the path.

$$
P_{L}^{A T C}=\min \left\{P_{1}^{A T C} / P T D F_{1}, \cdots, P_{n}^{A T C} / P T D F_{n}\right\}
$$

In this formula, $P_{n}^{A T C}$ indicates the tie-line / section corresponding to the nth gate on the transmission path $L$.

For the case of multiple transaction paths, the transaction space between the two provinces can not be obtained by directly summing the ATC of multiple paths. Therefore, based on the available transmission capacity and distribution coefficient of each tie-line / section in the transmission path between different networks provided by the power dispatching organization, this paper establishes the trading space analysis model for multiple transaction paths, and calculates the transaction space between the determined transmission and receiving end or the determined transmission tie-line in a certain period.

When the sending and receiving end is specified, the sending end is designated as area I, and the receiving end is designated as area III. The path set between the sending end and the receiving end is $L_{S D}$, and each transaction path is the series connection of multiple transmission tie-lines, so as to calculate the maximum power delivered by the sending end or purchased by the receiving end at a certain time or period. The model can be expressed as:

(1) Objective function

$$
\max P_{S}
$$

Or

$$
\max P_{D}
$$

(2) Constraint

$$
\begin{gathered}
P_{D}=P_{S} \cdot \prod_{l \in L}\left(1-\rho_{l}^{\text {loss }}\right) \\
P_{l}=P_{l-1} \cdot\left(1-\rho_{l-1}^{\text {loss }}\right), l \in L_{S D}^{m} \\
P_{l}^{A T C_{-}} \leq \sum_{n \in N} P T D F_{l n} \cdot P_{n} \leq P_{l}^{A T C_{+}}, l \in L_{S D}^{m}
\end{gathered}
$$

Where, $P_{S}$ is the demand of electricity generation, $P_{D}$ is the demand of electricity consumption, $\rho_{l}^{\text {loss }}$ is the network loss rate of tie-line $l, L_{S D}^{m}$ is the route $\mathrm{m}$-th in path set, $P T D F_{l n}$ is the power transmission distribution factor of node $\mathrm{n}$ injection tie-line $l, P_{n}$ is the injection power of node $\mathrm{n}, P_{l}^{A T C_{-}}$is the reverse transmission capacity of tie-line $l, P_{l}^{A T C_{+}}$is the forward transmission capacity of tie-line $l$.

\section{Case analysis}

\subsection{Analysis of daily stability limit}

Using MAPE and $\Delta$, the data of the same month in the two versions of daily stability limit data provided by the power dispatching organization are compared. The contact line where the trading space increases are found, as shown in the Fig.1. 


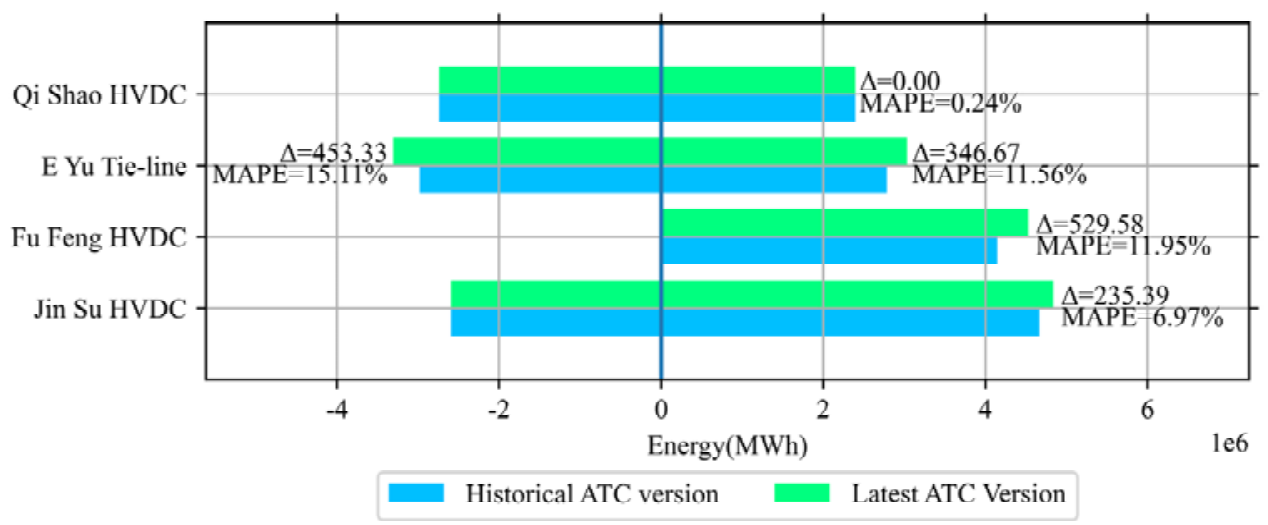

Figure 1. Comparison of daily stability limit ratio

As shown in Figure 1, the left side of the abscissa is the reverse limit quantity of electricity, and the right side is the forward limit quantity of electricity. It can be found that there are space release in the $\mathrm{E} \mathrm{Yu} \mathrm{Tie-line,}$ Jin Su HVDC, Qi Shao HVDC Tie-line and Fu Feng HVDC Tie-line. If the forward limit of Qi Shao HVDC is 0 , but MAPE is not equal to 0 , the total available transmission capacity will not change, but there are differences in daily stability limit in some time, such as

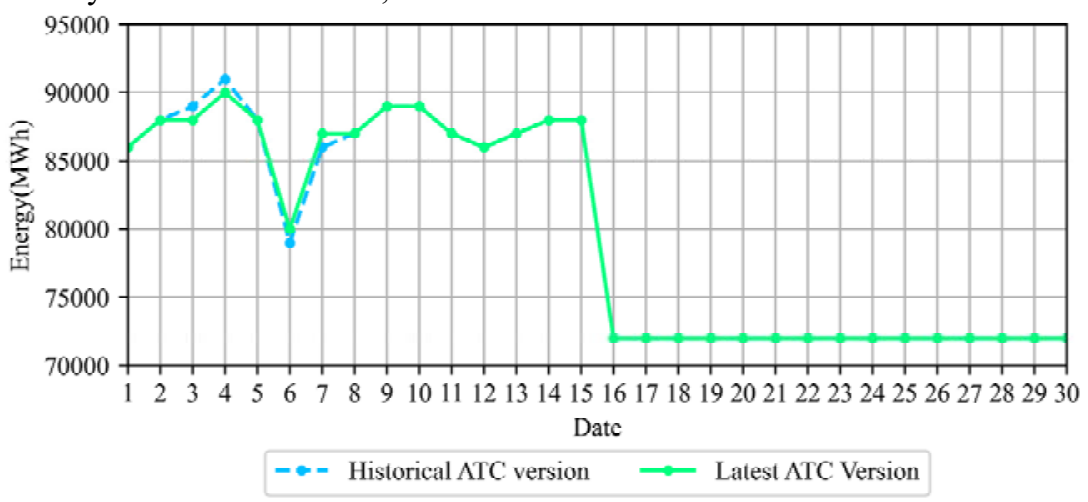

Figure 2. Comparison of Qi Shao HVDC daily stability limit ratio

\subsection{Analysis of trading space}

According to equations $(13) \sim(16)$, and referring to the line loss rate of inter-regional tie-line in the price scheme of cross provincial and cross regional trading power grid company of State Grid Corporation of China in 2020, this paper analyzes the trading space of available adjustment of maintenance plan but unchanged maintenance affected capacity, replacement of medium and long-term contracts, etc. The daily limit quantity of electricity of Qi Shao HVDC in this month is shown in Fig.3. It can be seen that the daily limit quantity of electricity increases on the 3rd and 4th days, but the total amount of monthly limit quantity of electricity remains unchanged. 


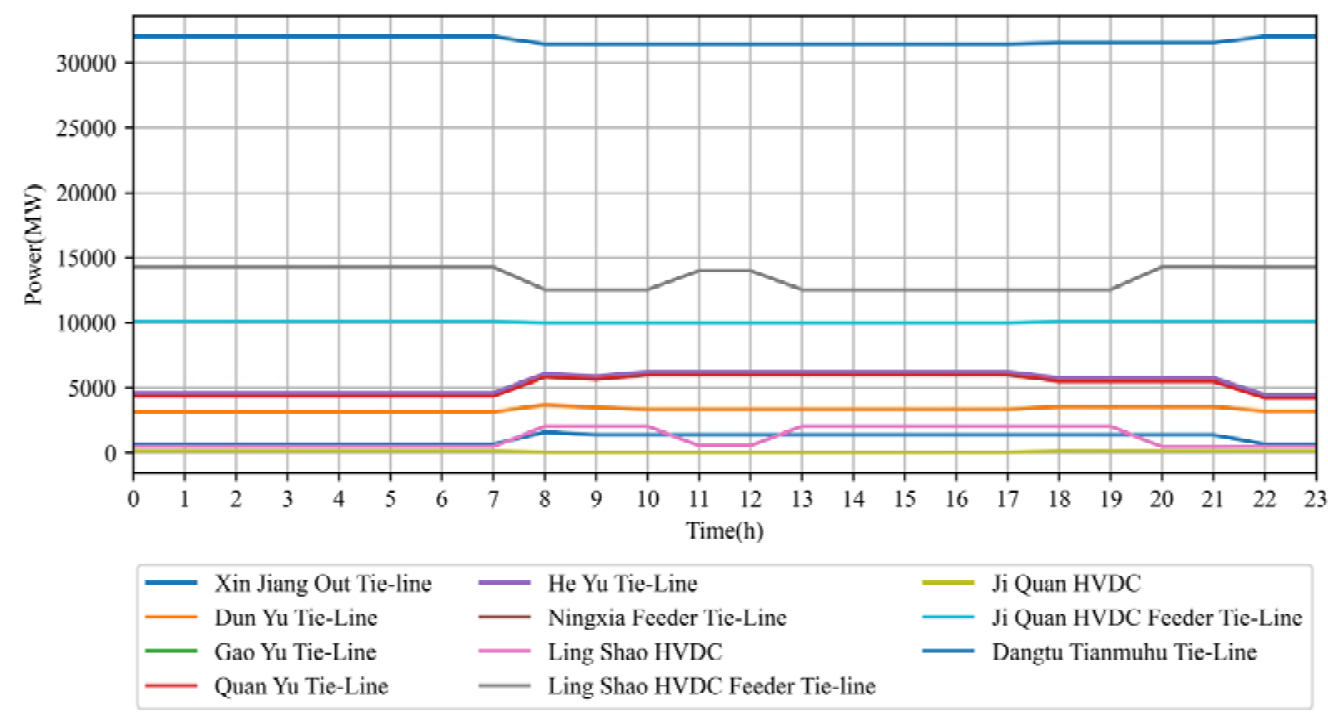

Figure 3. The available transmission capacity of Xinjiang's transmission channels to Jiangsu on a certain day

Fig. 3 shows the available transmission capacity of all tie-lines with distribution coefficient greater than 0.3 on that day. Fig.4 shows the maximum transaction volume of each tie-line on that day. The width of each section in the figure represents the transaction space size of the tieline; Table 3 shows the calculation result.

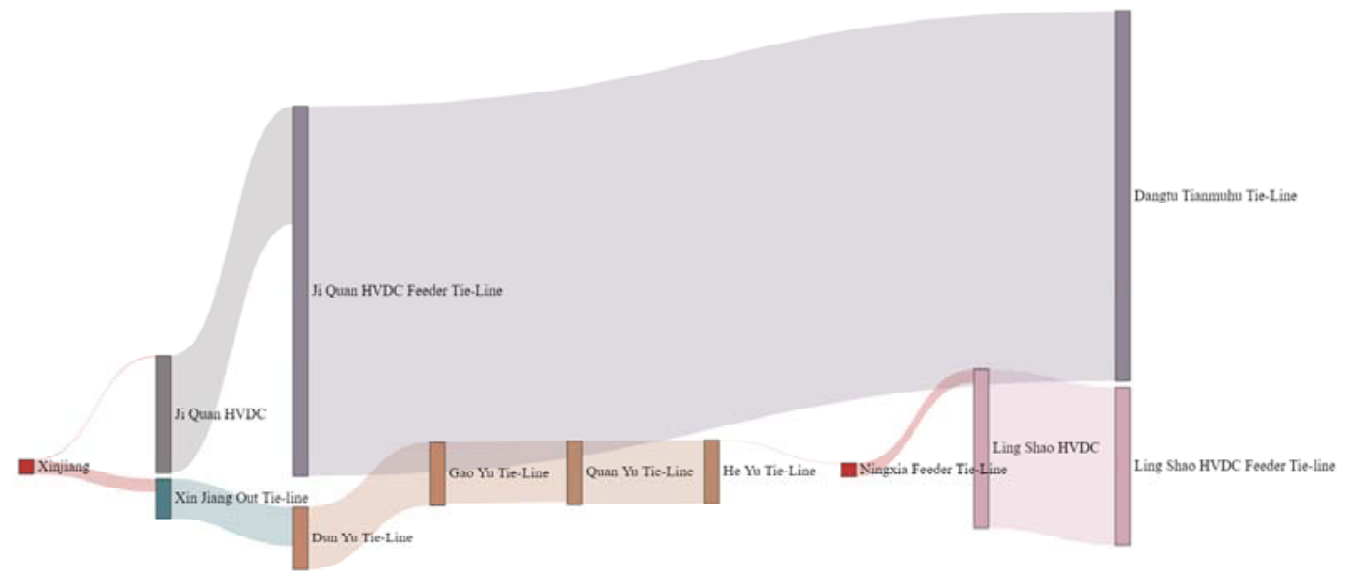

Figure 4. Transaction space analysis of Xinjiang's transmission channels to Jiangsu

Table 3. Transaction space calculation results of Xinjiang's transmission channels to Jiangsu

\begin{tabular}{ccc}
\hline Path & Tie-line Name & Transaction Space (MWh) \\
\hline \multirow{2}{*}{ Path I } & Xinjiang outer & 26748.00 \\
& Dun Yu & 80041.20 \\
& Gao Yu & 129149.20 \\
& Quan Yu & 124829.20 \\
& He Yu & 131309.20 \\
Ningxia feeder & 54024.00 \\
Ling Shao HVDC & 27524.00 \\
Path II & Ling Shao Receiving & 325042.24 \\
& Ji Quan HVDC & 2720.00 \\
& Ling Shao Receiving & 241664.00 \\
\hline
\end{tabular}

After analysis, there are two paths form Xinjiang to Jiangsu. The maximum trading space of Path $\mathrm{I}$ is determined by Ling Shao HVDC tie-line, and the maximum trading space of Path II is determined by $\mathrm{Ji}$ Quan HVDC tie-line. The trading space of the Path I is 27524 MWh and the trading space of Path II is 2720 MWh. If no trading path is specified, the maximum 
trading space between Xinjiang and Jiangsu is 30244MWh.

\section{Conclusion}

In this paper, a comparative analysis method is proposed for the available transmission capacity of main tie-line and daily stability limit data provided by power dispatching organization in inter-regional power trading, which is applied to the current national unified power market technical support platform and the new generation of power trading platform. A new method about analysis of different versions ATC data is came up by using the selection of data evaluation index, so as to promote the consumption of clean energy. At the same time, the transmission capacity can be used as a constraint to avoid the risk of congestion and reduce the possibility of safety check overrun. The related functions are applied in the national unified power market technical support platform and the new generation of power trading platform, which effectively supports the development of inter-regional power trading.

\section{Acknowledgments}

The authors would like to thank the State Grid Electric Power Research Institute, NARI Group Corporation, and NARI Technology Co., Ltd. for funding Project "Development and Application of Trading with Power Curve in Medium and Long-term Transaction Linkage with Spot Market”.

\section{References}

1. Central Committee of the Communist Party of China, State Council. Several Opinions on Further Deepening the Reform of the Electricity System (Zhongfa [2015] No. 9) [Z].2015.Beijing Power Exchange Center. Beijing Power Exchange 2019 Annual Report[R].Beijing:Beijing Power Exchange Center,2020.

2. LI Guodong, LI Gengyin, ZHOU Ming,et al.Analysis on Foreign Typical Market Mechanisms for Promoting New Energy Consumptive[J]. Electric Power,2019,52(02):46-52+60.

3. National Development and Reform Commission, National Energy Administration. Basic Rules for Medium- and Long-term Trading of Electricity (NDRC Energy Regulation [2020] No. 889). 2020.

4. HAN Tong, CHEN Yanbo, HAN Zijiao, et al. Optimal Power Flow Based Total Transfer Capability Calculation for AC/DC System with VSC-HVDC $[J]$. Automation of Electric Power Systems,2016,40(17):113-121.

5. XU Shaoxiang, MIAO Shihong, ZHOU Liangsong. Calculation Method of Available Transfer Capability for Cross-regional Interconnected Power Grid[J]. Automation of Electric Power Systems, 2017, 41(15):74-80+135.
6. ZHU JinzhouZHANG YanWANG Saiyi, et al. Probabilistic Optimal Decision-Making for Total Transfer Capability Under Electricity Market[J]. Power System Technology,2017,41(07):2282-2294.

7. ZHOU Lin, FU Xueqian, LIU Shuo, et al.DayAhead Market Clearing Model of Integrated Energy System for Promoting Renewable Energy Consumption[J]. Electric Power, 2019,52(11):9-18.

8. ZENG Dan, YANG Zhenglin, FENG Shuhai, et al, Inter-provincial Power Exchange Optimization Modeling Considering ATC Constrains of Hybrid AC/DC Power System[J].Power System Technology, 2020,44(10):3893-3899.

9. ZENG Dan, XIE Kai, PANG Bo,et al,Key Issues of National Unified Electricity Market with Chinese Characteristics (3): Research on Transaction Clearing Models and Algorithms Adapting to the Coordinated Operation of Provincial Electricity Markets[J].Power System Technology, 2020, 44(08):2809-2819.

10. Reliability E. Available Transfer Capability Definitions and Determination[J]. North American Electric Reliability Council Princeton New Jersey, 1996.

11. LI Xiaogang, YANG Libing, WU Min, et al.The Coordination Method and Its Characteristics of Two-Level Electricity Market[J]. Electric Power, 2020,53(09):71-80+116.

12. HU Chaofan, WANG Yang, ZHAO Tianyang, et al.Challenges and Strategies of Cross-provincial Intraday Market Design[J]. Power System Technology, 2020,44(02):573-579.

13. YAN Yu, LI Gengyin, LI Guodong, et al. Analysis of Power Direct Trade Organization Under New Round of Electricity Reform[J]. Electric Power, 2017,50(07):33-37.

14. XIE Kai. Electricity Market, Operation and Regulation: US Practice[M].Beijing:China Electric Power Press,2017. 